\title{
Fragmentation of Heavy Quarks Using Wave Function at the Origin
}

\author{
G. Rboroun ${ }^{1, *}$, H. Abdolmaleki ${ }^{2}$ \\ ${ }^{1}$ Physics department, Razi University, Kermanshah 67149, Iran \\ ${ }^{2}$ Department of Physics, Toyserkan branch, Islamic Azad University, Toyserkan, Iran \\ *Corresponding Author: boroun2001@yahoo.com
}

Copyright $(\subseteq 2013$ Horizon Research Publishing All rights reserved.

\begin{abstract}
In this paper we investigate the fragmentation function for heavy quarkanium using a global potential model. We employ the invariant vertex factor under parity, Lorentz transverse and invariant amplitude scattering for the fragmentation function. In the heavy quark limit we have obtained the S-wave analytical fragmentation functions for the heavy mesons at leading order perturbative QCD. The wave function of the bound state for mesons was set at origin of the framework. The fragmentation function is calculated with respect to the $\mathrm{Z}$ and Pt variables for $m_{1}+m_{2}$ and $M_{\text {experimental }}$ scales. In order to examine the accuracy of our calculations, the obtained results compared to the BFGW method.
\end{abstract}

Keywords Fragmentation, $J / \Psi$, WFO, Global Potential

\section{Introduction}

Light quarks fragmentation has been studied in various models [1]. These models include statistical manner as if the fragmentation parameter increase we observe that the fragmentation function decreases. After $\mathrm{b}$ and $\mathrm{c}$ quarks were discovered, the fragmentation mechanism of these quarks was described by Peterson in a quantum-mechanical model [2]. Another models used both on experimental and theoretical approaches $[3,4]$.

Many models have been used to describe the experimental fragmentation function that the most popular are the power and the Peterson models [1]. In applied theoretical model the wave function at origin describe the heavy quark system in limited range of hadron. Here, in order to avoiding the limitation of nucleus size of hadrons, we have used invariant amplitude and wave function at origin using a global potential.

\section{The Wave Function at Origin (WFO)}

The wave function at origin that has been calculated by the Schrödinger equation is one of the proper tools for calculating of fragmentation function [5]. These calculations are done in perturbation QCD that quarks and gluons are fixed with photons.

Indeed, for explain of the fragmentation function in our desired scale, we can used the Altarelli- Parisi evolution equations and also the wave function at the origin $|R(0)|$. As the ground state of heavy $\mathrm{q}$ and ${ }^{-} \mathrm{q}$ changes in non-relativity form, as systems including massive quarks would construct bounded states. So, for studying massive hadrons, evaluation of the Schrödinger equation is very important. In previous work, the potential provided by the Martin, logarithmic, Cornell, Lichtenberg ...

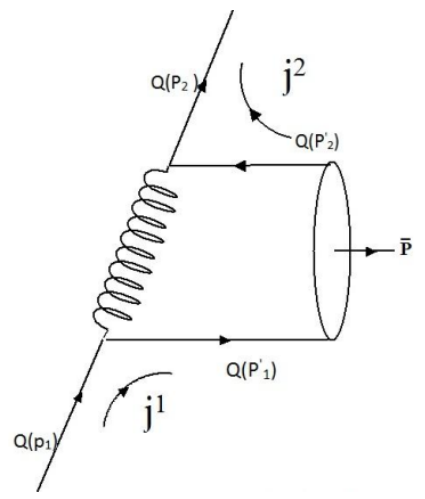

Figure 1. massive meson production

The Feynman diagram for this massive meson production is shown in Fig.1 at leading order (LO). Here we would like to consider the process of a heavy quark fragmentation with respect to a wave function at the origin (WFO). New chances have been brought up to apply non-relativity methods that are successful for bounded states in high-energy physics, which have been considered in the discovery of massive flavor mesons. So, for studying these mesons, evaluation of the Schrödinger equation is very important [6]. For calculate of the WFO, we used a global potential [5] in the following form, as:

$$
\begin{aligned}
& V(r)=k\left(\sqrt{r}+\frac{\alpha}{r}\right)+c \\
& k=070585, \alpha=0.46122, c=8.81715
\end{aligned}
$$


The constants can be determined when we fit method with the experimental data. The wave function at the origin with values calculated using the exact solution of the Schrödinger equation for the potential of the ground state is listed in Table 1 .

Table 1. The numerical values of the radial wave function at the origin, $|R(0)|^{2}=|\Psi(0)|^{2} / 4 \pi$, for 1S, 2S, 2P state of $b \bar{b}, c \bar{c}, b \bar{c}$ system with respect to the global potential in reference [5].

\begin{tabular}{|c|c|c|c|}
\hline & $1 \mathrm{~s}\left(\mathrm{GeV}^{3}\right)$ & $2 \mathrm{~s}\left(\mathrm{GeV}^{3}\right)$ & $2 \mathrm{p}\left(\mathrm{GeV}^{3}\right)$ \\
\hline$|R(0)|^{2}$ for $\quad b \bar{b}$ & 5.97601 & 5.98 & 2.7042 \\
\hline$|R(0)|^{2}$ for $c \bar{c}$ & 0.50414 & 0.50366 & 0.02670 \\
\hline$|R(0)|^{2}$ for $b \bar{c}$ & 1.21126 & 1.21078 & 0.267802 \\
\hline
\end{tabular}

\section{Fragmentation function}

In the Parton model each particle carry out, $X_{i}$ fraction of the momentum-energy of meson with the constraint of $\sum_{i} X_{i}=1$

.In the infinite four- momentum frame, the output four-momentum of meson is $\bar{P}$ which produces a jet. Then the four- momentum particles have these momentums as:

$$
P_{1 \mu}^{\prime}=X_{1} \overline{\mathrm{P}} \quad, \quad P_{2 \mu}^{\prime}=X_{2} \overline{\mathrm{P}} \text {. }
$$

Therefore we can write for each particle four- momentums in the following form:

$$
\begin{aligned}
& P_{1 \mu}^{\prime}=X_{1} Z P_{1 \mu}, \quad P_{2 \mu}^{\prime}=X_{2} Z P_{1 \mu} \\
& P_{2 \mu}=(1-Z) P_{1 \mu}
\end{aligned}
$$

Where we used the fragmentation parameter, $\mathrm{z}$, as given by,

$$
Z=\frac{P_{\text {Hadron }}}{P_{\text {Quark }}}=\frac{\bar{P}}{P_{i}}
$$

With the choice of the wave function at origin according to a global potential that is obtained from [14], the fragmentation function for massive quark has the following general form, as:

$$
D\left(z, \mu_{0}\right)=\sum_{n} d_{n}\left(z, \mu_{0}\right)\left\langle o_{n}^{x}\right\rangle
$$

where $d_{n}\left(z, \mu_{0}\right)$ gives the probability for the Parton $i$ to form a jet that includes a $c \bar{c}$ pair in the state labeled by n, and $\left\langle\begin{array}{l}x \\ n\end{array}\right\rangle$ is proportional to the probability for a point like $c \bar{c}$ pair in the $\mathrm{n}$ - state. According to the current- current interaction and hard amplitude scattering [9], the probability $\left\langle o_{n}^{x}\right\rangle$ given by the following form:

$$
\begin{aligned}
& \left\langle o_{n}^{x}\right\rangle=\int d^{3} P_{1}^{\prime} d^{3} P_{2}^{\prime} d^{3} P\left|T_{H}\right|^{2} W F O \\
& T_{H}=T_{Q \rightarrow M}=\frac{C_{f} g^{2}{ }_{s} m_{1} m_{2}}{\sqrt{2 p_{01}^{\prime} p_{02}^{\prime} p_{02} p_{01}}} \frac{\delta^{3}\left(p_{1}^{\prime}+p_{2}^{\prime}+p_{2}-p_{1}\right)}{\left(p_{01}^{\prime}+p_{02}^{\prime}+p_{02}-p_{01}\right)}(W F O) \overline{\mathfrak{M}} \\
& \left\langle o_{n}^{x}\right\rangle=\left(C_{f} g^{2}{ }_{s} m_{1} m_{2}(W F O)\right)^{2} \\
& \quad \int d^{3} P_{1}^{\prime} d^{3} P_{2}^{\prime} d^{3} P_{2} \times \frac{1}{2 p_{01}^{\prime} p_{02}^{\prime} p_{02} p_{01}} \frac{\delta^{3}\left(p_{1}^{\prime}+p_{2}^{\prime}+p_{2}-p_{1}\right)}{\left(p_{01}^{\prime}+p_{02}^{\prime}+p_{02}-p_{01}\right)^{2}}|\overline{\mathfrak{M}}|^{2}
\end{aligned}
$$


Here $T_{H}$ is the hard scattering amplitude.

Also the unpolarized fragmentation that average over initial quark spin and sum over final state particles has the following form for invariant amplitude:

$$
\begin{aligned}
& |\overline{\mathfrak{M}}|^{2}=1 / q^{4} L_{Q}^{\mu v} L_{\mu v}^{\bar{Q}} \\
& L_{Q}^{\mu v}=1 / 2 \sum_{s p i n}\left(\bar{U}\left(p_{1}\right) \gamma^{\mu} U\left(p_{1}^{\prime}\right)\right)\left(\bar{U}\left(p_{1}\right) \gamma^{\mu} U\left(p_{1}^{\prime}\right)\right)^{*} \\
& L_{\mu v}^{\bar{Q}}=1 / 2 \sum_{s p i n}\left(\bar{U}\left(p_{2}\right) \gamma^{\mu} V\left(p_{2}^{\prime}\right)\right)\left(\bar{U}\left(p_{2}\right) \gamma^{\mu} V\left(p_{2}^{\prime}\right)\right)^{*} \\
& |\overline{\mathfrak{M}}|^{2}=\frac{8}{q^{4}}\left[\left(p_{1}^{\prime} \cdot p_{2}\right)\left(p_{1} \cdot p_{2}^{\prime}\right)+\left(p_{1}^{\prime} \cdot p_{2}^{\prime}\right)\left(p_{1} \cdot p_{2}\right)\right]
\end{aligned}
$$

Where, $L_{Q}^{\mu v}$ is invariant under proper Lorentz transformations and parity operation. Therefore the invariant amplitude in the infinite momentum limit can be written:

$$
|\overline{\mathfrak{M}}|^{2}=\frac{8}{q^{4}}\left[\left(p_{1}^{\prime} \cdot p_{2}\right)\left(p_{1} \cdot p_{2}^{\prime}\right)+\left(p_{1}^{\prime} \cdot p_{2}^{\prime}\right)\left(p_{1} \cdot p_{2}\right)\right]
$$

The dot products in Eq.6 are given by:

$$
\begin{aligned}
& 2\left(P_{1}^{\prime} \cdot P_{2}\right)=\frac{1-\mathrm{Z}}{\mathrm{X}_{1} \mathrm{Z}}\left(\mathrm{m}_{1}^{2}\right)+\frac{\mathrm{X}_{1} \mathrm{Z}}{1-\mathrm{Z}}\left(\mathrm{m}_{2}^{2}+\mathrm{P}_{\mathrm{T}}^{2}\right) \\
& 2\left(P_{1} \cdot P_{2}^{\prime}\right)=\frac{1}{\mathrm{X}_{2} \mathrm{Z}}\left(\mathrm{m}_{2}^{2}\right)+\mathrm{X}_{2} \mathrm{Z}\left(\mathrm{m}_{1}^{2}+\mathrm{P}_{\mathrm{T}}^{2}\right) \\
& 2\left(P_{1}^{\prime} \cdot P_{2}^{\prime}\right)=\frac{\mathrm{X}_{1}}{\mathrm{X}_{2}}\left(\mathrm{~m}_{2}^{2}\right)+\frac{\mathrm{X}_{2}}{\mathrm{X}_{1}}\left(\mathrm{~m}_{1}^{2}\right) \\
& 2\left(P_{1} \cdot P_{2}\right)=1-\mathrm{Z}\left(\mathrm{m}_{1}^{2}+\mathrm{P}_{\mathrm{T}}^{2}\right)+\frac{1}{1-\mathrm{Z}}\left(\mathrm{m}_{2}^{2}+\mathrm{P}_{\mathrm{T}}^{2}\right)-2 \mathrm{P}_{\mathrm{T}}^{2}
\end{aligned}
$$

Where $\mathrm{X}_{2}=\mathrm{X}=1-\mathrm{X}_{1}$.

Therefore, the fragmentation function for heavy meson with WFO of global potential [5] is obtained from equations $(5,6)$, as we have:

$$
\begin{aligned}
D\left(z, \mu_{0}\right)= & \left(P_{q q}+P_{q g}\right)\left(C_{f} g_{s}^{2} m_{1} m_{2}(W F O)\right)^{2} \\
& \sum \int d^{3} P_{1}^{\prime} d^{3} P_{2}^{\prime} d^{3} P_{2} \times \frac{1}{2 p_{01}^{\prime} p_{02}^{\prime} p_{02} p_{01}} \frac{\delta^{3}\left(p_{1}^{\prime}+p_{2}^{\prime}+p_{2}-p_{1}\right)}{\left(p_{01}^{\prime}+p_{02}^{\prime}+p_{02}-p_{01}\right)^{2}}|\overline{\mathfrak{M}}|^{2}
\end{aligned}
$$

Where $P_{q q}, P_{q g}$ are the functions obtained from [10]. In order to solving integrals in phase space, we can integrate into two integrals elements. Thus we obtain the solution of phase space integrals according to the following equations, as:

$$
\begin{aligned}
\int d^{3} \bar{P} & \frac{\delta^{3}\left(\bar{p}_{0}+p_{2}-p_{1}\right)}{p_{01}\left(\bar{p}_{0}+p_{02}-p_{01}\right)^{2}}=\frac{p_{01}}{\left(\bar{p}+p_{2}\right)^{4}} \\
& \int f\left(z, P_{T}\right) d^{3} P_{2}=\int f\left(z, P_{T}\right) d P_{2 L} d^{2} P_{T} \\
& =x^{2} M^{2} p_{01} f\left(z,\left\langle P_{T}\right\rangle^{1 / 2}\right)
\end{aligned}
$$


In the infinite momentum limit, the integration from longitudinal momentum convert to energy integration and also transverse integration convert to average transverse momentum, respectively. This means simply replace $P_{T}$ with its average value. Finally, we have

$$
\begin{aligned}
& D(Z)=\left(\frac{Z\left(P_{q q}+P_{q g}\right)}{(1-X)(1-Z) M^{3}}\left(32 \pi^{4}\right)\left(C_{f} g_{s}^{2}(W F O) M X\right)^{2}\left((1-Z) X^{2} M^{2}\right)^{3}\right) \frac{|\overline{\mathfrak{M}}|^{2}}{\xi^{2} \Lambda^{2}} \\
& \Lambda=X Z\left(\mathrm{~m}_{1}^{2}+\mathrm{P}_{\mathrm{T}}^{2}\right)\left(\frac{2-Z}{1-Z}\right)+\mathrm{m}_{1}^{2}\left(\frac{1}{X Z}+\frac{1-X}{X Z}+\frac{X}{1-X}+\frac{1-X}{X}+X^{2} Z^{2}\right) \\
& \xi=q^{2}=\frac{\left(m_{1}^{2}\right)+X^{2} Z^{2}\left(m_{1}^{2}+\left(P_{T}\right)^{2}\right)}{X Z}
\end{aligned}
$$

This equation defines the fragmentation function for $J / \psi$ meson.

\section{Result and Discussion}

The explicit form of Eq.(11) in our calculations, for $J / \psi$ meson at two case $\mathrm{M}=\mathrm{m}_{1}+\mathrm{m}_{2}$ and $\mathrm{M}=\mathrm{M}_{\text {exp. }}$, is given by the following form, respectively, as:

$$
\begin{aligned}
& D(Z)=0.9062\left(\frac{4\left(1+(1-z)^{2}\right)}{3 z}+\frac{4\left(1+z^{2}\right)}{3(1-z)}\right) \times \frac{K}{F} \\
& K=\left(3.7968(1-z)^{2}+4.309 z^{2}-0.9742(1-z) z^{2}+4.309(1-z)^{2} z^{2}+0.5433 z^{4}\right) \\
& F=\left(z\left(1.9485(1-z)^{2}+2.43567(1-z) z+0.7371 z^{2}\right)^{2}\right. \\
& \left.\quad \times\left(1.9485+0.4871 z^{2}-\frac{2\left(1.9485(1-z)^{2}+0.7371 z^{2}\right)}{(1-z) z}\right)^{2}\right)
\end{aligned}
$$

and

$$
\begin{aligned}
D(Z)=0.5927\left(\frac{4\left(1+(1-z)^{2}\right)}{3 z}+\frac{4\left(1+z^{2}\right)}{3(1-z)}\right) \times \frac{K}{F} \\
K=\left(3.7968(1-z)^{2}+4.0678 z^{2}-0.7917(1-z) z^{2}+4.6338(1-z)^{2} z^{2}+0.5327 z^{4}\right) \\
F=\left(z\left(1.9485(1-z)^{2}+2.8568(1-z) z+0.7299 z^{2}\right)^{2}\right. \\
\left.\quad \times\left(1.9485+0.5878 z^{2}-\frac{1.8206\left(1.9485(1-z)^{2}+0.8895 z^{2}\right)}{(1-z) z}\right)^{2}\right)
\end{aligned}
$$

Firstly we examined the behavior of Eq.11 with respect to Pt scales. Secondly, in figure 2, the result appears in various values ranging from 3-100 GeV for heavy quark fragmentation. Then we compared our fragmentation functions with the experimental predications [11]. Therefore our comparison shows that the fragmentation functions in high Pt scale have same procedure with the experimental predicate [11]. Therefore in Fig.3 we compared our fragmentation functions with BFGW predication [11]. 


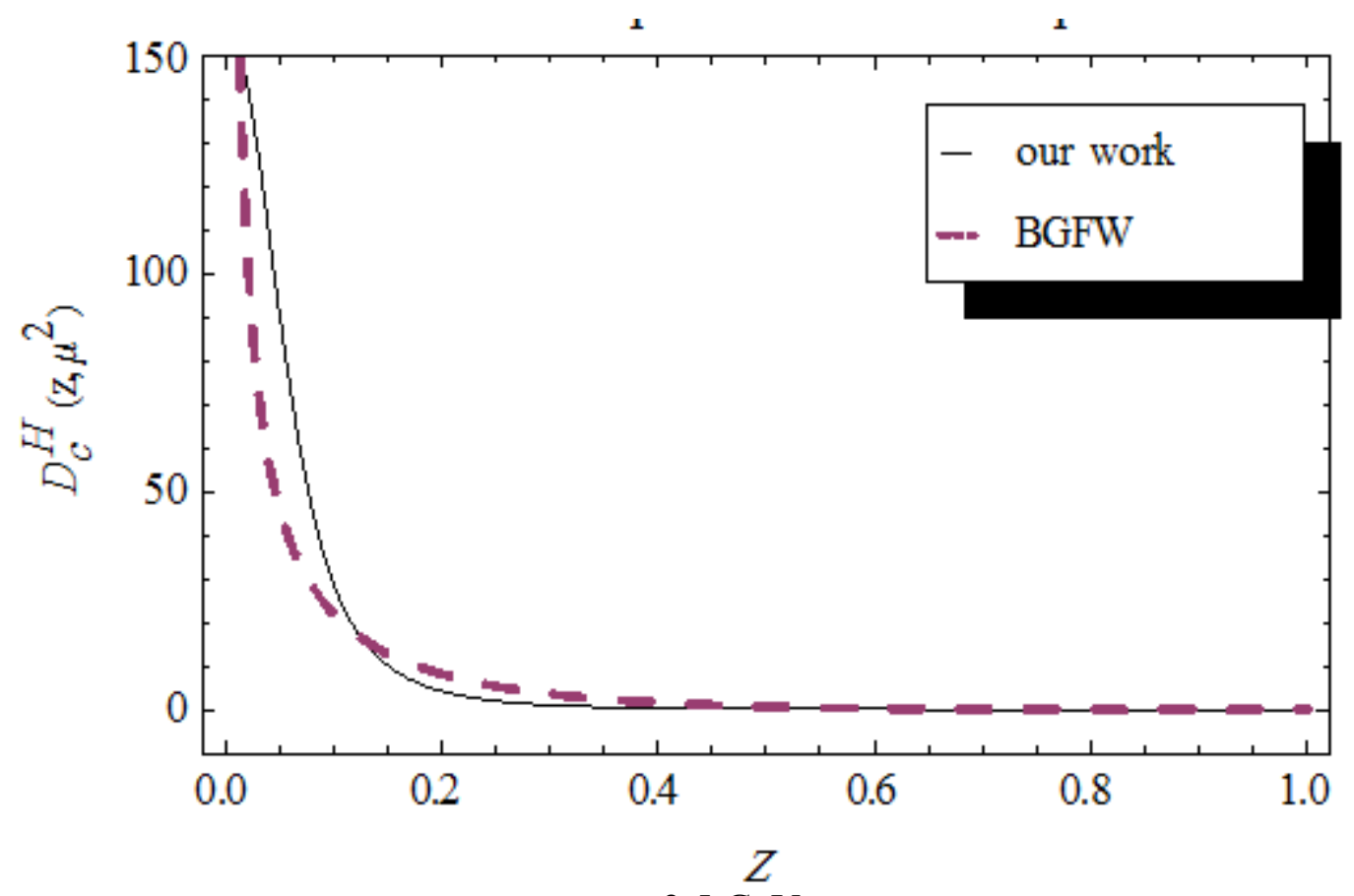

Figure 2a. Our Fragmentation function according to $\mathrm{z}$ for $\mu_{0}=3.5 \mathrm{GeV}$ scale compared with BFGW predication model (dash line)

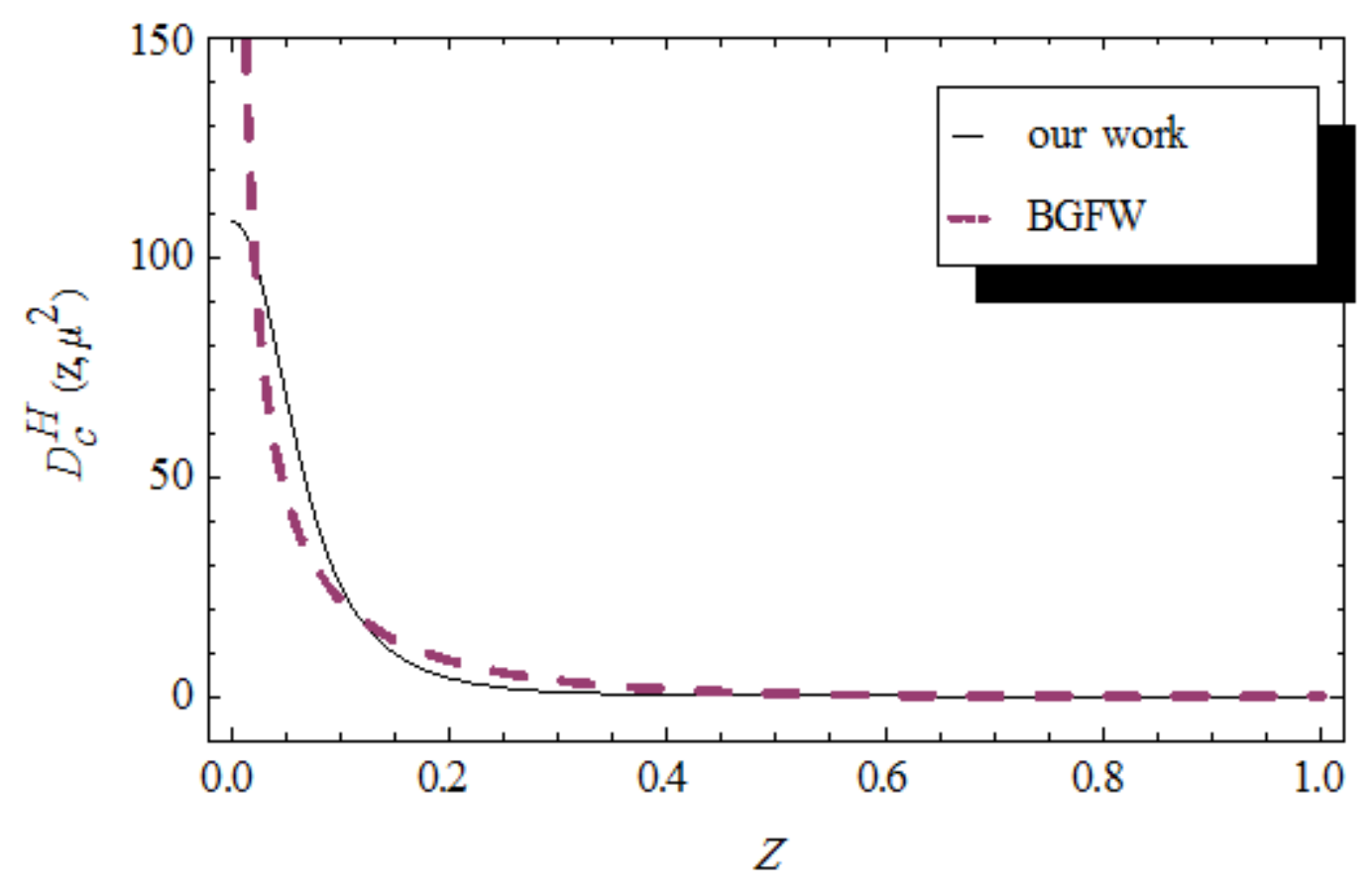

Figure 2b. The same as Fig.2-a for $\mu_{0}=3.09691 \mathrm{GeV}$ scale 


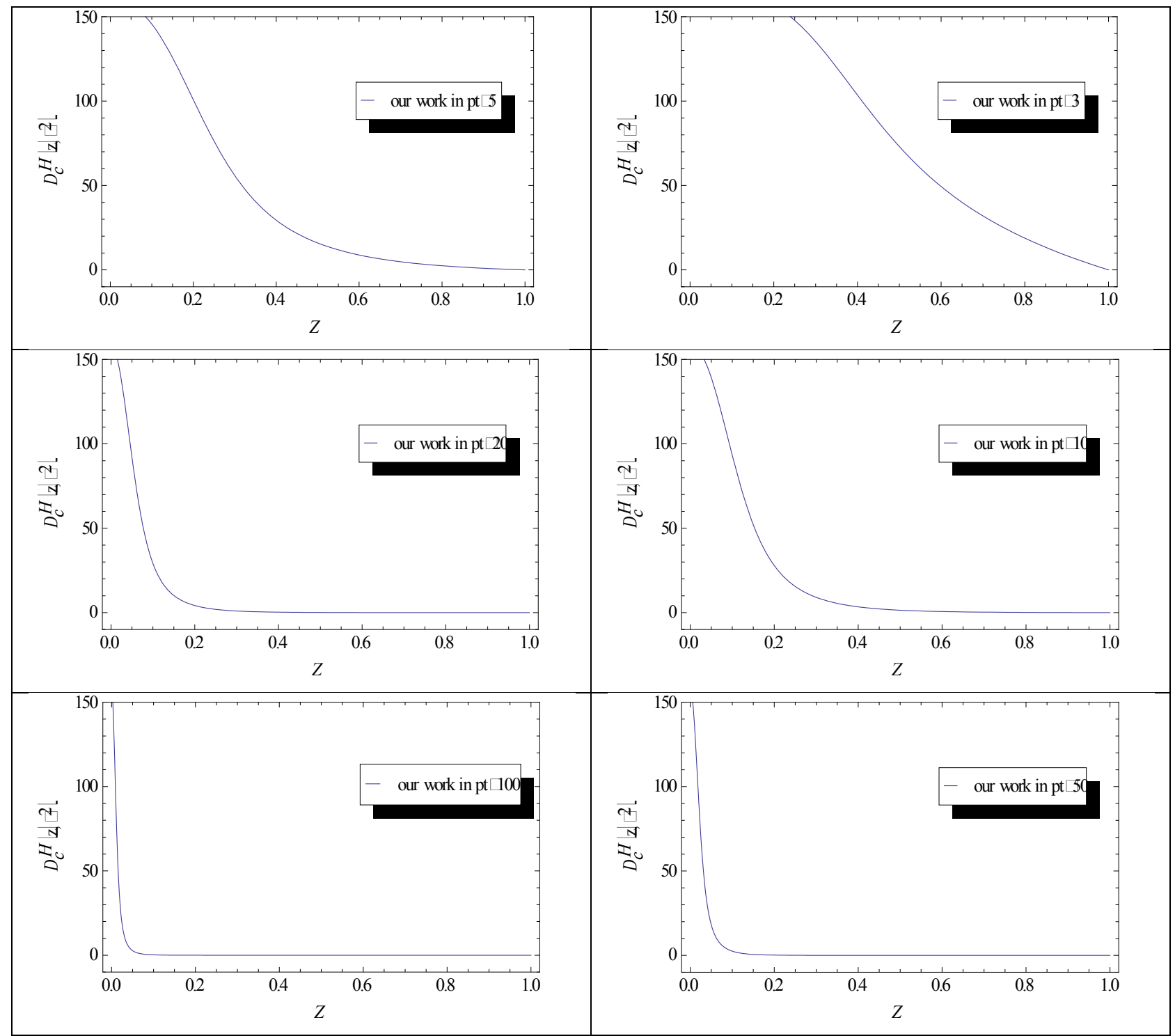

Figure 3. Fragmentation functions at different $\mathrm{pt}(\mathrm{GeV})$ scales

\section{Conclusion}

In the heavy quark limit we have obtained the exact analytical fragmentation functions for S-wave two heavy mesons at leading order perturbative QCD. The wave function at the origin (WFO) for bound state wave function of mesons has been used. In this model, the $\mathrm{C}$ quark mass is considered to be $1.3959 \mathrm{Gev}$. Fragmentation functions have been calculated for LEP energy. In this determination we used the vertex invariant under parity, Laurence transfer, and hard scattering amplitude for heavy mesons.

\section{REFERENCES}

[1] B. Andersson et. al., Phys. Rept. 97 (1983) 31 B, R. Webber Nucl. Phys. B 238 (1984)492

[2] C. Peterson et. al., Phys. Rev. D 27(1983) 105

[3] E.L.Berger and D.Jones, Phys.Rev.D23(1981) 1521 S.D.Ellis, M.Einhorn and C.Quigg, Phys.Rev.Lett.36 (1976) 1263 M.Cacciari and M.Greco, Phys.Rev.Lett.(1994)1586; K.Sridhar, A.D.Martin and W.J.Stirling, phys.lett.B438(1998)211; A.Petrell, Nucl.phys.Proc.Supp.86(2000)533. FredCooper, et.al., arXiv:hep-ph/0402219v2(2004)

[4] C.Albajar, et.al.,phys.lett.B256(1991)112; F.Abe, et.al.,Phys.Rev.Lett.69(1992)3704 F.Abe, et.al.,Phys.Rev.Lett.71(1993)2537; RaphaelGranierde, Cassagnac, PHENIXCollaboration,J.Phys.G: Nucl.and Part.Phys.30(2004)S1341;

[5] G. R. Boroun and H. Abdolmalki, Phys. Scr. 80 (2009) 065003 . 
[6] C.Quigg and J.L.Rosner, Phys. Rep. 56,(1979) 167.

[7] Braaten E and Cheung K 1995 Phys. Rev. D 514819

[8] E.Eichten and C.Quigg, Phys. Rev. D 49, (1994) 5845.
[9] M.A. GomshiNobary and B. Javadi, Eur.Phys.J.C42,(2005)37.

[10] G. C. Nayak, arXiv:hep-ph/0905.3922v2,(2009).

[11] L .Bourhis,et. al,. arXiv:hep-ph/0009101v1 (2000) 